\title{
Band gap determination of Ni-Zn ferrites
}

\author{
G P JOSHI, N S SAXENA*, R MANGAL, A MISHRA ${ }^{\dagger}$ and T P SHARMA \\ Department of Physics, University of Rajasthan, Jaipur 302 004, India \\ ${ }^{\dagger}$ Department of Physics, C.C.S. University, Meerut 250 004, India
}

MS received 22 January 2003; revised 9 April 2003

\begin{abstract}
Nanocomposites of Ni-Zn with copolymer matrix of aniline and formaldehyde in presence of varying concentrations of zinc ions have been studied at room temperature and normal pressure. The energy band gap of these materials are determined by reflection spectra in the wavelength range $400-850 \mathrm{~nm}$ by spectrophotometer at room temperature. From the analysis of reflection spectra, nanocomposites of copolymer of aniline and formaldehyde with $\mathrm{Ni}_{1-x} \mathrm{Zn}_{x} \mathrm{Fe}_{2} \mathrm{O}_{4}(x=0 \cdot 0,0 \cdot 2,0 \cdot 4,0 \cdot 6,0 \cdot 8$ and 1.0) have been found to have direct band gaps ranging from 1.50-1.66 eV.
\end{abstract}

Keywords. Nanocomposite; optical properties; reflection spectra.

\section{Introduction}

In the past few years nanocomposites have become one of the most extensively studied materials all over the world as they possess several applications such as magnetic recording materials, sensors etc (Anderson et al 1980). The nanomaterials can be polycrystalline in nature and may belong to inorganic, organic or a combination of both classes of materials (Vadera et al 1997; Mathur et al 1999). Moreover, nanocomposite materials composed of oxides and conducting polymers have brought out more fields of applications such as smart windows, magnetic refrigeration at high temperature, high-density information storage, colour imaging, ferrofluids, medical diagnosis, electromagnetic wave absorption, toners in photocopying, conductive paints, drug delivery, rechargeable batteries etc (Suri et al 2001). Materials with nanoscale microstructure, i.e. nanocrystalline materials or nanocomposites, are increasingly becoming important for their technological importance due to their unique electrical, magnetic and optical properties. They can be distinguished from the nanocrystalline and nanophase materials on the basis that in case of nanocrystalline materials only one phase exists. While in the case of nanomaterials more than one Gibbson solid phases are present and out of which at least one of the phases is in the nanometer size range (Komaneni 1992). Earlier (Joshi et al 2002), energy band gap studies of these materials have been reported using absorption spectra. However, using thick pellets the effect of scattering in the absorption spectra cannot be properly estimated, as for computing the energy band gap, we could never experimentally

*Author for correspondence obtain $(\alpha h v)^{2}=0$ (Singh et al 2000). This problem can be solved by studying reflection spectra, where the exact value of energy band gap can be obtained as we may have $(\alpha h v)^{2 / 3}=0$. The reflection spectra of nanocomposite materials in the wavelength range 850-400 nm (Kumar et al 1999) have been used to determine the band gap.

\section{Experimental}

Nanocomposites of $\mathrm{Ni}-\mathrm{Zn}$ ferrite in a copolymer matrix of aniline and formaldehyde were synthesized at room temperature by using a novel chemical method reported elsewhere (Mathur et al 1998). The nanocomposites of $\mathrm{Ni}_{1-x} \mathrm{Zn}_{x} \mathrm{Fe}_{2} \mathrm{O}_{4}$ ferrites with $x=0.0,0 \cdot 2,0.4,0 \cdot 6,0.8$ and 1.0 were synthesized in a copolymer matrix containing three different monomers of aniline and formaldehyde (ortho, para and meta). As a typical preparation, sample S1 $(x=0)$ was synthesized by treating the aqueous solution of aniline $(0 \cdot 10 \mathrm{~mol})$, hydrochloric acid $(0 \cdot 12 \mathrm{~mol})$, formaldehyde $(0 \cdot 10 \mathrm{~mol})$ with an aqueous solution of halides of nickel $(0 \cdot 189 \mathrm{~mol})$ taken according to the stoichiometry. The resulting solution was stirred thoroughly and added to $10 \%$ solution of $\mathrm{NaOH}$. The precipitated composite was washed repeatedly with distilled water till the filtrate was free of alkali $(\mathrm{pH} \mathrm{7.5)}$ and then dried in air. Similarly, the samples S2-S6 $(x=0.2,0.4,0.6,0.8$ and 1.0$)$ were synthesized using the same procedure by varying the quantities of nickel and zinc according to the stoichiometry. The reflection spectra of nanocomposites of $\mathrm{Ni}-\mathrm{Zn}$ ferrite in a copolymer matrix of aniline and formaldehyde in presence of varying concentrations of zinc ions in bulk form were taken by spectrophotometer Hitachi model U-3400 at room temperature. 


\section{Characterization of sample}

The energy band gap of these materials is determined by the reflection spectra. According to the Tauc relation, the absorption coefficient, $\alpha$, for direct band gap material is given by (Sirohi and Sharma 1999)

$$
\alpha h v=A\left(h v-E_{\mathrm{g}}\right)^{n},
$$

where $E_{\mathrm{g}}$ the energy gap, constant $A$, is different for different transitions, $(h v)$ is energy of photon and $n$ is an index which assumes the values $1 / 2,3 / 2,2$ and 3 depending on the nature of the electronic transition responsible for the reflection. Also absorption coefficient, $\alpha$, is directly proportional to $\ln \left\{\left(R_{\max }-R_{\min }\right) /\left(R-R_{\min }\right)\right\}$ and is given by

$$
2 \alpha t=\ln \left[\left(R_{\max }-R_{\min }\right) /\left(R-R_{\min }\right)\right],
$$

where $t$ is the thickness of the sample, $R_{\max }$ and $R_{\min }$ are maximum and minimum values of reflectance, $R$ the reflectance at a given photon energy, $h v$. To understand the nature of energy band gap transition in these materials, a graph of $\ln \left[h v \ln \left\{\left(R_{\max }-R_{\min }\right) /\left(R-R_{\min }\right)\right\}\right]$ vs $\ln (h v-$ $\left.E_{\mathrm{g}}\right)$ is drawn for the case of sample $\mathrm{S} 6$ as shown in figure 1. The plot in the figure is a straight line, the slope of which gives $n=3 / 2$. This confirms that the transition is a forbidden direct transition in these materials.

When graph is plotted between $(\alpha h v)^{2 / 3}$ or $[h v \ln$ $\left.\left\{\left(R_{\max }-R_{\min }\right) /\left(R-R_{\min }\right)\right\}\right]^{2 / 3}$, and $h v$ (as abscissa), a straight line is obtained. The extrapolation of the straight line to $(\alpha h v)^{2 / 3}=0$ axis gives the value of the band gap of the sample.

\section{Results and discussion}

Figure 2 shows the reflection spectra of nanocomposites of $\mathrm{Ni}_{1-x} \mathrm{Zn}_{x} \mathrm{Fe}_{2} \mathrm{O}_{4}$ (with $x=0 \cdot 6$ ). It is observed from figure 2 that reflection decreases with the decrease in

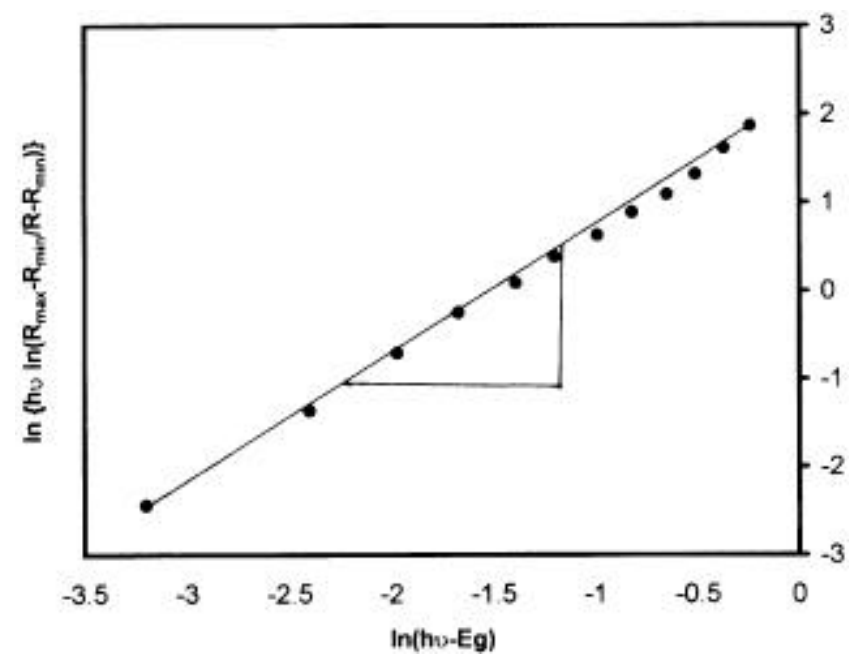

Figure 1. Plot of $\ln \left\{h v \ln \left(R_{\max }-R_{\min } / R-R_{\min }\right)\right\}$ vs $\ln (h v-$ $E_{\mathrm{g}}$ ) (Tauc 1974). wavelength. A sudden decrease at a particular wavelength, indicates the presence of optical band gap in these samples. These nanocomposites of $\mathrm{Ni}-\mathrm{Zn}$ with copolymer matrix of aniline and formaldehyde in presence of varying concentrations of zinc ions are direct band gap materials and therefore the Tauc relation (Tauc 1974) as given in (1) is used for the determination of direct band gap in the nanocomposites of $\mathrm{Ni}_{1-x} \mathrm{Zn}_{x} \mathrm{Fe}_{2} \mathrm{O}_{4}$. Graphs between $\left[h v \ln \left\{\left(R_{\max }-R_{\min } / R-R_{\min }\right)\right\}\right]^{2 / 3}$ vs $h v$ have been plotted in figure 3 . The extrapolation of

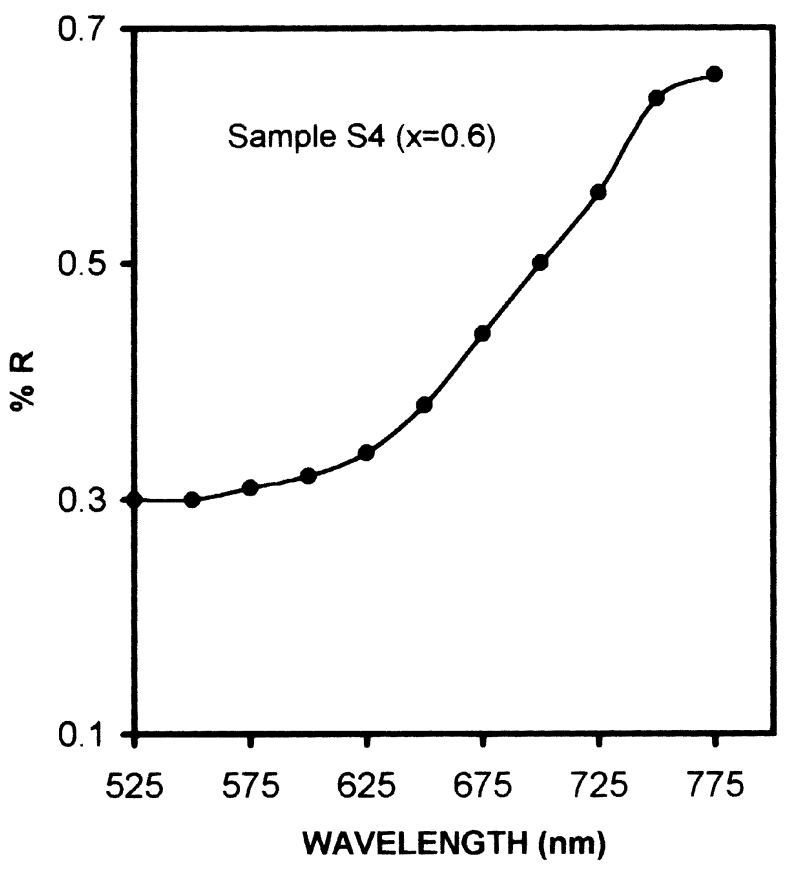

Figure 2. Reflection spectra of sample $\mathrm{S} 4(\bullet) \mathrm{Ni}_{0 \cdot 4} \mathrm{Zn}_{0 \cdot 6} \mathrm{Fe}_{2} \mathrm{O}_{4}$.

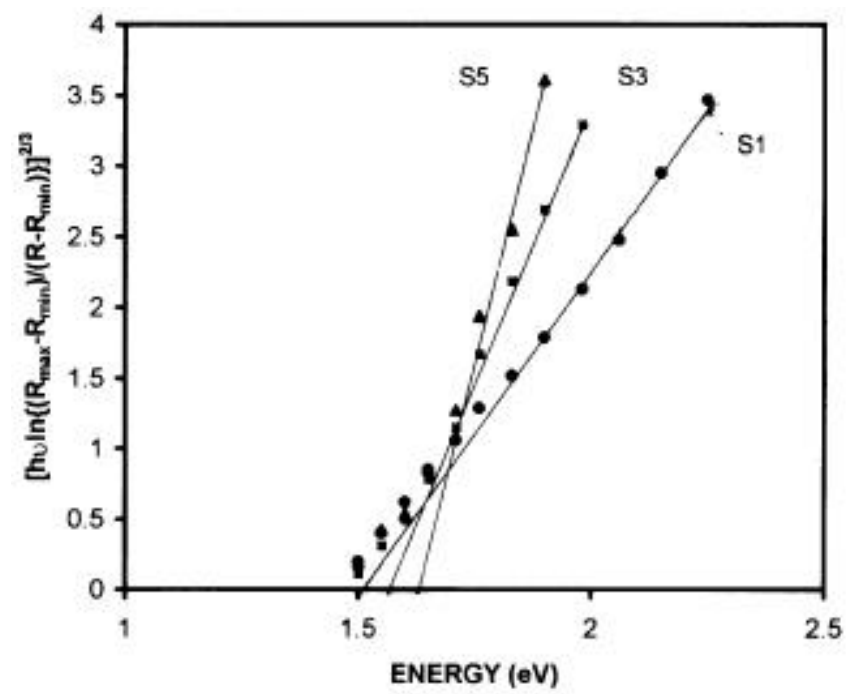

Figure 3. Band gap determination of sample $\mathrm{S} 1(\bullet) \mathrm{Ni}_{1 \cdot 0^{-}}$ $\mathrm{Zn}_{0.0} \mathrm{Fe}_{2} \mathrm{O}_{4}$; sample $\mathrm{S} 3(-) \mathrm{Ni}_{0.6} \mathrm{Zn}_{0.4} \mathrm{Fe}_{2} \mathrm{O}_{4}$ and sample S5 $\mathrm{Ni}_{0.2} \mathrm{Zn}_{0.8} \mathrm{Fe}_{2} \mathrm{O}_{4}$. 


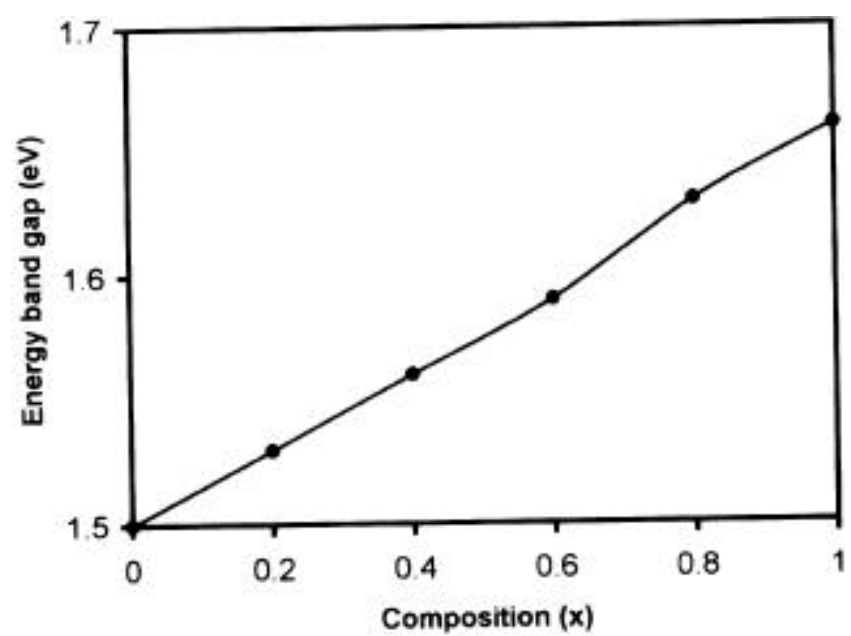

Figure 4. Band gap vs composition.

straight line to $[(\alpha h v)]^{2 / 3}=0$ gives the value of energy band gap. From these graphs, the value of energy band gap of sample $\mathrm{S} 1$ (composite of $\mathrm{Ni}_{1-x} \mathrm{Zn}_{x} \mathrm{Fe}_{2} \mathrm{O}_{4}$ with $x=0 \cdot 0), \mathrm{S} 3(x=0 \cdot 4)$ and $\mathrm{S} 5(x=0 \cdot 8)$ comes out to be $1.50,1.56$ and $1.63 \mathrm{eV}$, respectively. Similarly, for samples S2 (with $x=0 \cdot 2$ ), S4 (with $x=0 \cdot 6$ ) and S6 (with $x=1 \cdot 0$ ), the obtained values of optical band gap are 1.53 , 1.59 and $1.66 \mathrm{eV}$, respectively. The variation of optical band gap with $x$ (different compositions) is given in figure 4 . It can be observed from figure 4 that optical band gap increases continuously $(x=0 \cdot 0-1 \cdot 0)$ with the increase of $\mathrm{Zn}$ concentration in the nanocomposites of $\mathrm{Ni}_{1-x} \mathrm{Zn}_{x} \mathrm{Fe}_{2} \mathrm{O}_{4}$. In some of the ternary semiconductors i.e. $\operatorname{InAs}_{x} \mathrm{P}_{1-x}$, it has been observed that energy band gap decreases with increase in the lattice parameter. Therefore, when we consider the variation of energy band gap with concentration of $\mathrm{Zn}$ in the above case, the increase in energy gap value with the increase of $\mathrm{Zn}$ concentration could be explained on the basis of decrease in lattice parameter due to increase in $\mathrm{Zn}$ concentration.

\section{Conclusions}

The experimentally observed band gap increase with the increase in the concentration of $\mathrm{Zn}$ suggests that the structural change occurring in the composite is responsible for such a variation.

\section{References}

Anderson A, Hunderi O and Granqvist C G 1980 J. Appl. Phys. 57757

Joshi G P, Mangal R, Saxena N S and Sharma T P 2002 Indian J. Pure \& Appl. Phys. 40297

Komaneni S 1992 J. Mater. Chem. 212

Kumar V, Sharma S K, Sharma T P and Singh V 1999 Opt. Mater. 12115

Mathur R, Sharma D R, Vadera S R and Kumar N 1999 Bull. Mater. Sci. 22991

Mathur R, Parihar M, Vadera S R and Kumar N 1998 J. Magn. Soc. Jap. 22273

Singh V, Singh B P, Kumar V, Sharma T P and Tyagi R C 2000 Indian J. Eng. \& Mater. Sci. 7100

Sirohi S and Sharma T P 1999 Opt. Mater. 13267

Suri K, Annapoorni S and Tandon R P 2001 Bull. Mater. Sci. 24563

Tauc J 1974 Amorphous and liquid semiconductor (New York: Plenum) p. 159

Vadera S R, Mathue R, Parihar M and Kumar N 1997 NanoStructured Mater. 8889 